\title{
INHERITANCE OF GROWTH RATE IN NEUROSPORA CRASSA: REVERSE SELECTION IN AN IMPROVED STRAIN
}

\author{
K. E. PAPA,* A. M. SRB and W. T. FEDERER \\ Department of Plant Breeding, Cornell University, Ithaca, New York
}

Received 6.ix.66

\section{INTRODUCTION}

A CLASSical method of determining limits to selection responses has been to measure response when selection is applied in the opposite direction. If genotypic fixation of the selected lines has occurred, then response in either direction will not be possible unless new genetic variation arises or is induced. Numerous studies on the nature of selection limits and related phenomena have been conducted on a variety of organisms. In many instances genetic variability still existed in selected lines, yet additional selection responses were not realised. Presumably, this genetic variability could not contribute to further selection responses. Experimental evidence in support of this notion has been offered and encompasses various phenomena.

In certain instances, natural selection in the form of differential fertility was found to be opposing artificial selection (Falconer, I955; Lee and Pateman, 196I). A. Robertson (1955) reported that the maintenance of high genetic variance, although response to selection had ceased, could occur as a result of a negative correlation between the character selected and fitness components. Clayton and A. Robertson (1957) attributed unfixable genetic variation in selected lines to the selection of heterozygotes for a lethal gene. The possibility that a physiological limit may set upper limits on a selected character was examined by Falconer and King (1953). On crossing individual strains of mice which presumably had reached selection limits, they observed a renewed response to selection for increased and decreased body size.

Lines having reached apparent selection limits due to the absence of free genetic variability might still possess potential genetic variability. Utilisation of this potential genetic variability, however, would depend on breaking up rather tight linkages to get recombinant individuals of superior phenotype (Mather, I943). Theoretical treatments of linkage and selection, although quite complex, have been partially examined by Fraser (1957, 1960) using Monte Carlo methods.

Recent quantitative inheritance studies in various Neurosporas have shown linear growth rate to be under the control of several to many genes (Papa, Srb and Federer, I966). The slow and gradual increase in growth rate during artificial selection was indicative of a polygenic

* Present address: Department of Plant Pathology and Plant Genetics, University of Georgia, Athens. 
system. In addition, fitting the selection data to a theoretical statistical model, which assumed that a large number of genes controls growth rate, resulted in relatively good correspondence between observed and expected responses (Federer, Papa and Srb, 1966). Asymptotic upper limits predicted by the model appeared to have been reached. In an effort to determine whether limits had been reached in selecting for fast growth rate, different selection procedures were initiated. The present paper presents results obtained from the study of a line which had previously ceased to respond to further selection pressure.

\section{MATERIALS AND METHODS}

The sources of the various strains of Neurospora, cultural conditions, statistical analyses and the techniques involved in the selection programme have been given previously (Papa et al., I 966) and will be reviewed only briefly here. Neurospora strains utilised in the present study included one from Honduras (Hon3A) and one of the standard St Lawrence strains of $\mathcal{N}$. crassa (77a). Standard techniques for crossing and making isolations were used (Beadle and Tatum, 1945). All crosses were made at $25^{\circ} \mathrm{C}$. Cultures were grown and maintained on a complete medium, whereas growth rates were determined on a minimal medium. Growth rates were measured in specially constructed growth tubes. Methods used for preparing and handling growth tubes were those described by Ryan et al. (1943).

Selection for fast growth rate was practised among the progeny of the cross Hon3A/77a.* Sixty ascospores were isolated at random and from the resulting vegetative cultures 40 were randomly selected and tested for mating type. Ten cultures of each mating type were randomly selected. Conidia from each were transferred into duplicate growth tubes and incubated at $35^{\circ} \mathrm{C}$. The fastest growing two progeny of opposite mating type were selected and crossed, eventually giving rise to progeny which were subjected to another cycle of selection in exactly the manner already outlined. Second samples taken from the original cross and subsequent cycles of selection constituted a second replication.

Considerable progress was made in selecting for fast growth rate. An apparent cessation of response, however, was noted after approximately nine generations of selection. Since the lack of response was accompanied by an almost complete lack of free genetic variability, a new selection procedure (fig. I) was initiated. Following I I cycles of selection for fast growth in one replication, single growth rate measurements were taken at $35^{\circ}$ on 40 vegetative cultures (20 of each mating type).

Crosses between the fastest of each mating type, the slowest of each mating type and crosses between the fastest and slowest of opposite mating types were made to give three new generations. The selection pressure previously being $0 \cdot 1$ was now changed to 0.05 in order to see whether response might be renewed in the fast $\times$ fast line and whether, if it were possible to back select, this might be accomplished in fewer generations.

Forty progeny (20 of each mating type) from each of the three new generations were then tested at the same time, using the same media at $35^{\circ} \mathrm{C}$. The fastest of each mating type in the fast $\times$ fast line were crossed. Similarly, the fastest and slowest in the fast $\times$ slow line and the slowest in the slow $\times$ slow line were crossed. This procedure was followed in the succeeding generations of selection. Corresponding generations of selection in each of the three lines were handled as similarly as possible in order that accurate comparisons could be made among progeny means of the three methods at each cycle.

After I 7 cycles of selection for fast growth rate, reverse selection was again

* Conidial and protoperithecial parent on left and right side of diagonal line, respec-
ely. tively. 
initiated in the fast $x$ fast line. The procedure involved was identical to the one described for reverse selection commencing at the IIth cycle. The four lines (fast $\times$ fast, fast $\times$ slow, and the two slow $\times$ slow) were treated as similarly as possible.

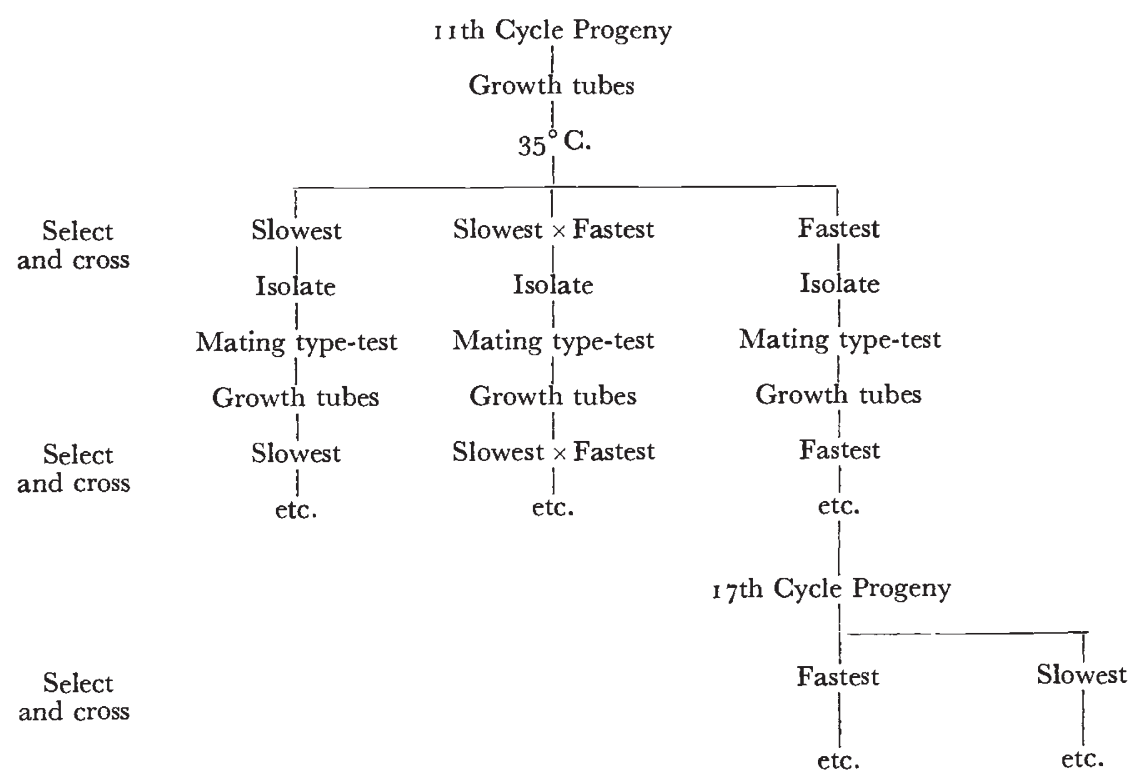

FIG. I.-The selection procedure.

Following six generations of reverse selection, crosses were made between progeny obtained by reverse selection and those arising after 17 cycles of selection for fast growth rate. Single growth tube measurements at $35^{\circ} \mathrm{C}$. were taken on 85 progeny from each cross. Second measurements were taken on the same progeny one month later. Measurements were also taken on ro vegetative transfers of the parents. The parents, randomly spaced among the progeny, were included only in the first set of measurements.

\section{RESULTS}

Response to selection for fast growth rate in the cross Hon $3 \mathrm{~A} / 77 \mathrm{a}$ at $35^{\circ}$ C. is portrayed in fig. 2. Responses from the first 10 generations were based on progeny means from two replications. Responses from subsequent generations, although based on the same number of progeny, were obtained from one replication. Genetic variances for the first ro generations are also included in fig. 2. Each variance represents the average of two replications.

Considerable progress was made in selecting for fast growth rate over the first five generations. From generation 5 to generation 10 little additional response was noted. The nature of this response corresponded quite well with the predicted response based on a theoretical model (Federer et al., 1966). An asymptotic upper limit predicted by the model was realised prior to generation 10 . The magnitude of the genetic variances between generations 5 and 10 indicates an almost complete lack of available genetic variability. 
Response to selection in the fast $\times$ slow line was no different from the response obtained in the fast $\times$ fast line (table $\mathrm{I}$ ) and was therefore not included in fig. 2. Since only single growth rate measurements were taken after generation Io, genetic variances could not be obtained. Among-progeny variances, however, are presented in table 2. They reflect the total variability in each generation.

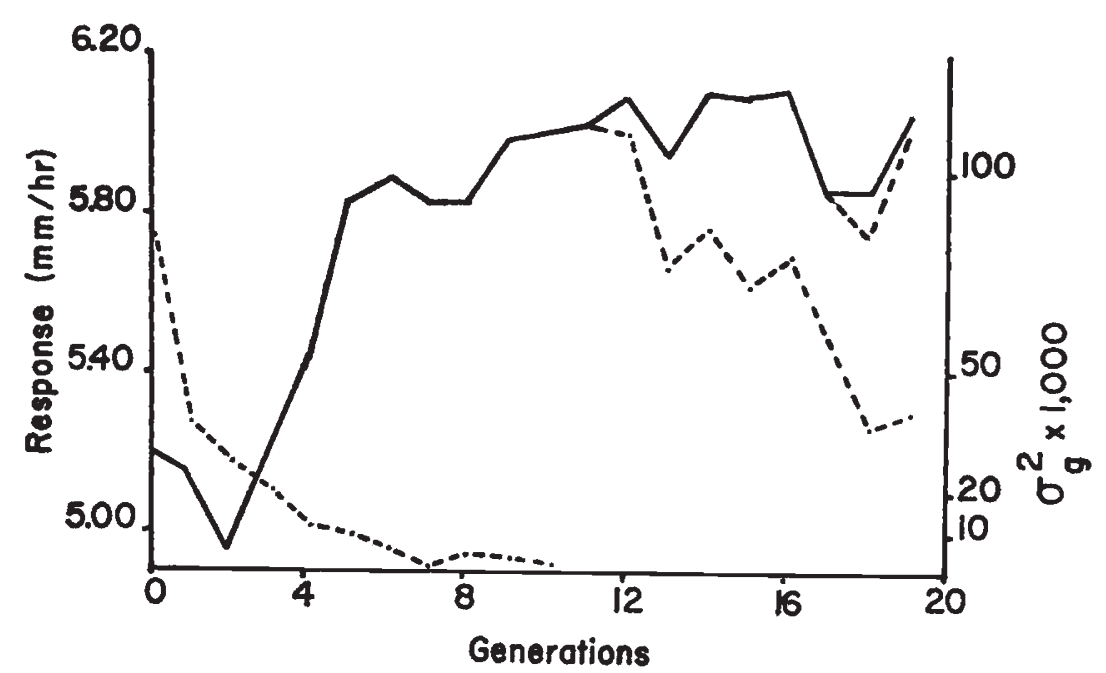

Fig. 2.-Response to selection for fast growth rate in the cross Hon $3 \mathrm{~A} / 77 \mathrm{a}$ at $35^{\circ} \mathrm{C}$. (solid line). Response from the first Io generations is based on progeny means of two replications. Reverse selection, initiated at generations I I and I 7 , is shown by broken lines. Genetic variances for cycles o-10 are connected by broken lines.

Essentially no difference was detectable in the amount of variation among progeny from the fast $\times$ fast and fast $\times$ slow selection lines (table 2). More total variability was noted in the slow $\times$ slow line, however, than in either of the other two selection lines for all generations except generation I4. This increased variability was particularly evident in generations $\mathrm{I} 2, \mathrm{I} 7$ and $\mathrm{I} 8$.

The first cycle of reverse selection resulted in a significantly slower growth rate than was achieved by selection for fast growth rate. The second cycle of reverse selection resulted in an even greater spread between either fast $\times$ fast or fast $\times$ slow and slow $\times$ slow selection lines. In addition to a difference in growth rate, a distinct morphological difference could be detected. Progeny resulting from reverse selection possessed a denser growth pattern with less conidiation than did progeny in either of the other two types of selection. A relatively constant difference in the pattern of growth, as well as in the rate of growth, was obtained between fast $\times$ fast and slow $\times$ slow selection lines until the I 7 th cycle of selection for fast growth (corresponding to the 6 th cycle of reverse selection). The progeny in the 6th cycle of reverse selection, however, were segregating for growth rate. Consequently, the mean growth rate was further reduced in the next generation of 
reverse selection. A small but statistically significant decrease in growth rate was obtained in the first cycle of reverse selection, which had been initiated following I 7 cycles of selection for fast growth rate. No additional decrease was observed in the second cycle.

TABLE I

Growth rate differences (mm./hr.) obtained by three methods of selection

\begin{tabular}{|c|c|c|c|c|}
\hline & \multicolumn{3}{|c|}{ Comparisons } \\
\hline & $(\mathbf{F} \times \mathbf{F})-(\mathbf{F} \times \mathbf{S})$ & $(\mathbf{F} \times \mathbf{F})-(\mathrm{S} \times \mathbf{S})^{11}$ & $(\mathbf{F} \times \mathbf{S})-(\mathrm{S} \times \mathrm{S})^{11}$ & $(\mathbf{F} \times \mathbf{F})-(\mathrm{S} \times \mathrm{S})^{17}$ \\
\hline & & & & \\
\hline I 2 & 0.04 & $0.09^{*}$ & $0.05^{*}$ & $\ldots$ \\
I 3 & -0.05 & $0.29^{*}$ & $0.34^{*}$ & $\ldots$ \\
I & 0.05 & $0.35^{*}$ & $0.30^{*}$ & $\ldots$ \\
I5 & 0.00 & $0.47^{*}$ & $0.47^{*}$ & $\ldots$ \\
I6 & 0.03 & $0.41^{*}$ & $0.8^{*}$ & $\ldots$ \\
I 7 & -0.03 & $0.40^{*}$ & $0.43^{*}$ & $0.11^{*}$ \\
I8 & 0.01 & $0.59^{*}$ & $0.8^{*}$ & $0.06^{*}$ \\
I9 & 0.02 & $0.75^{*}$ & $0.73^{*}$ & \\
\hline
\end{tabular}

* Denotes significance at the $0.01 / \mathrm{m}$. level of probability. A per experiment error rate with 3 or 4 (m.) comparisons (Federer, I96I).

$\mathbf{F}=$ fast, $S=$ slow, $(S \times S)^{11}=$ reverse selection begun at cycle $I$ i and $(S \times S)^{17}=$ reverse selection begun at cycle 17 .

The rather step-wise decrease in growth rate in the reverse selection line suggested that each step might correspond to the control of a single gene. In order to test this assumption, I 7 th cycle-progeny obtained from the slow $\times$ slow and fast $\times$ fast selection lines were

TABLE 2

Total variation among the progeny in each selection line from generations 12-19

\begin{tabular}{|c|c|c|c|c|}
\hline \multirow{2}{*}{ Cycle } & \multicolumn{4}{|c|}{ Selection line } \\
\hline & $(\mathbf{F} \times \mathbf{F})$ & $(\mathbf{F} \times \mathrm{S})$ & $(\mathrm{S} \times \mathrm{S})^{11}$ & $(S \times S)^{17}$ \\
\hline I 2 & 0.0053 & 0.0032 & 0.0150 & $\cdots$ \\
\hline I3 & $0.007 \mathrm{I}$ & 0.0058 & 0.0 I I I & $\cdots$ \\
\hline I 4 & 0.0152 & 0.0122 & 0.0055 & $\ldots$ \\
\hline I 5 & 0.0022 & 0.0027 & 0.0054 & $\cdots$ \\
\hline I 6 & 0.0025 & $0.002 \mathrm{I}$ & 0.0042 & $\ldots$ \\
\hline I 7 & 0.0050 & 0.0055 & 0.0228 & $\cdots$ \\
\hline I8 & $0.006 \mathrm{I}$ & 0.0171 & 0.0794 & 0.0064 \\
\hline I9 & 0.0029 & 0.0044 & 0.0069 & 0.0060 \\
\hline
\end{tabular}

crossed. Since the progeny from the 6th cycle of reverse selection were segregating for growth rate, two individuals arbitrarily chosen to represent two different levels of growth were crossed to the same individual from the fast $\times$ fast selection line. The progeny were placed into two classes depending on the pattern of growth, which was easily detectable in growth tubes. 
The upper distributions in fig. 3 represent the progeny from the cross between 2 IA (fast $\times$ fast line) and 26 a (slow $\times$ slow line). The progeny distribution on the left and shown by the heavy line represents progeny possessing a dense growth pattern with little conidiation. The progeny distribution on the right corresponds to progeny possessing a more sparse growth pattern with more conidiation. The parental

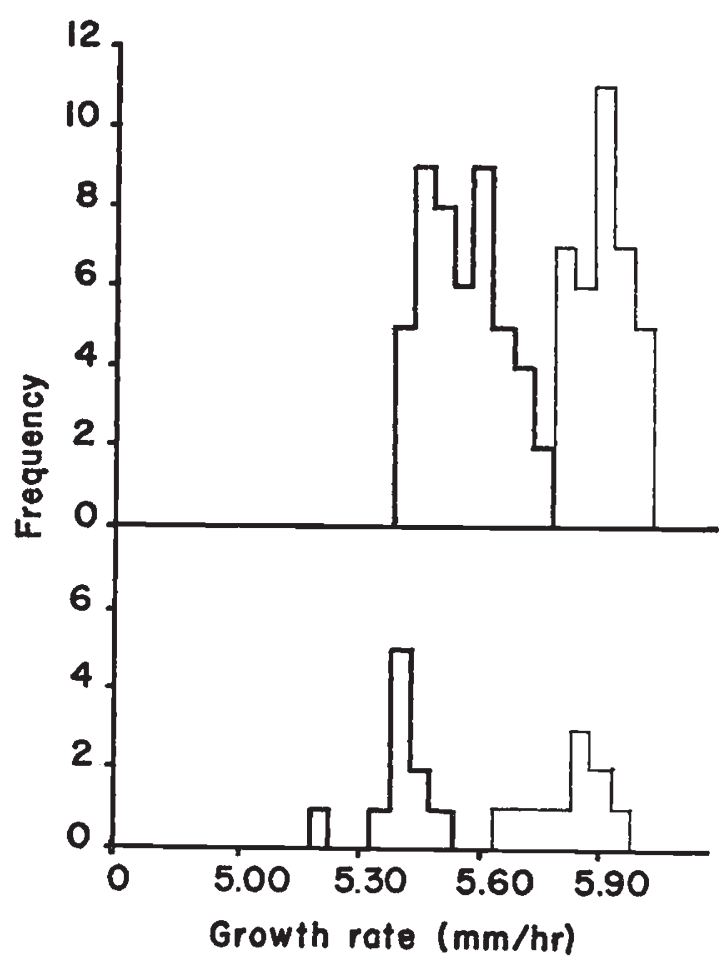

Frg. 3.-Frequency distributions of growth rates at $35^{\circ} \mathrm{C}$. for the parents $(26 \mathrm{a}$ and $21 \mathrm{~A})$ and their progeny. Parents and progeny are represented by lower and upper distributions, respectively. Dense growth pattern is indicated by heavy line, normal growth by light line.

distributions are shown in the lower portion of fig. 3. Culture $26 \mathrm{a}$, represented by the heavy line, had a mean growth rate of $5.46 \mathrm{~mm}$./ $/ \mathrm{hr}$. with a dense growth pattern. In comparison, $21 \mathrm{~A}$, represented by the distribution on the right, had a mean growth rate of $5.90 \mathrm{~mm}$./hr. and a sparse growth pattern.

The progeny were segregating in approximately a $\mathrm{I}:$ I ratio with reference to the pattern of growth. In addition, there appeared to be a close association between growth rate and pattern of growth. Two rather distinct distributions were obtained: one in which the progeny had a dense growth pattern and a mean growth rate of $5.64 \mathrm{~mm}$. $/ \mathrm{hr}$. and the other having sparse growth with a mean growth rate of $5.94 \mathrm{~mm}$./hr. Apparently, the dense pattern of growth had the effect of reducing growth rate by about $0.3 \mathrm{~mm}$. $/ \mathrm{hr}$. 
Distributions in fig. 4 represent the growth rates of the parents, 2IA and I5a (a full sib to 26a), and their progeny. Parental and progeny distributions are portrayed in the lower and upper portions of fig. 4, respectively. Culture $15 \mathrm{a}$, with an average growth rate of $4.92 \mathrm{~mm}$./hr., was significantly slower than $26 \mathrm{a}$. Both, however, possessed the same pattern of growth. The progeny were again

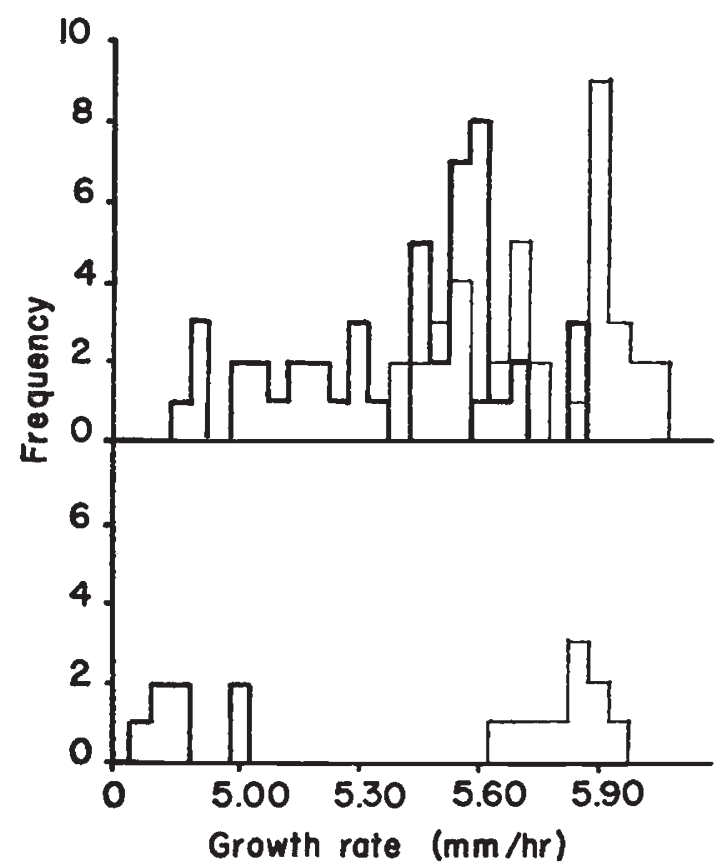

FIG. 4.-Frequency distributions of growth rates at $35^{\circ} \mathrm{C}$. for the parents ( $15 \mathrm{a}$ and $2 \mathrm{I} \mathrm{A}$ ) and their progeny. For explanation see fig. 3 .

segregating approximately in a ratio of $\mathrm{I}: \mathrm{I}$, with reference to the pattern of growth, thereby suggesting that a single gene was controlling this particular trait. Progeny scored on the basis of their growth pattern, however, could not be placed into two distinct distributions. Presumably the lack of distinct distributions was due to the segregation of other genes affecting growth rate.

A minimal estimate of the number of genes involved between these selected lines was calculated according to the method of Mather (r 949) when applied to haploids (Croft and Simchen, 1965; Simchen, I 966). This method assumes that all of the favourable $(+)$ alleles of the $k$ genes, whose differences are involved in the cross, are concentrated in one parent and all of the unfavourable (-) alleles in the other parent. It also assumes that each pair of alleles has an equal effect and that the genes are unlinked. Failure of these assumptions will cause an underestimate of $k$. The formula

$$
k=\frac{(\mathrm{I} / 2 \text { Parental mean difference })^{2}}{\hat{\sigma}_{g}^{2}}
$$


provides an estimate of the number of genes $(k)$ involved. $\hat{\sigma}_{g}^{2}$ is an estimate of the genetic variation among the progeny. Estimates of $k$, genetic and environmental components of variance and parental mean differences are presented in table 3. Mean square expectations are: error (date by progeny interaction), $\sigma_{e}^{2}$; among progeny, $\sigma_{e}^{2}+2 \sigma_{g}^{2}$. These mean squares, along with date of measurement mean square, are included in table 3 .

Highly significant differences were obtained between dates of measurements. Similar results were obtained by Papa (1964). Variation among multiple growth tube measurements on the same culture on the same date was generally quite small and nonsignificant whereas

TABLE 3

Analyses of variance of growth rates of progenies from crosses between selected lines, parental mean differences and estimates of the genetic variances and the number of genes (k)

\begin{tabular}{|c|c|c|c|c|c|c|c|c|c|}
\hline \multirow{2}{*}{ Cross } & \multicolumn{2}{|c|}{ Date } & \multicolumn{2}{|c|}{ Progeny } & \multicolumn{2}{|c|}{ Error } & \multirow{2}{*}{$\hat{\sigma}_{g}^{2}$} & \multirow{2}{*}{$\begin{array}{c}\text { Parental } \\
\text { mean } \\
\text { difference }\end{array}$} & \multirow{2}{*}{$\hat{k}$} \\
\hline & d.f. & M.S. & d.f. & M.S. & d.f. & M.S. & & & \\
\hline $\begin{array}{l}21 \mathrm{~A} \times 26 \mathrm{a} \\
2 \mathrm{~A} \times 15 \mathrm{I}\end{array}$ & I & $\begin{array}{l}0 \cdot 7692^{* *} \\
0 \cdot 7901 * *\end{array}$ & $\begin{array}{l}83 \\
84\end{array}$ & $\begin{array}{l}0.0576^{* *} \\
0 \cdot 1426^{* *}\end{array}$ & $\begin{array}{l}82 \\
84\end{array}$ & $\begin{array}{l}0 \cdot 0064 \\
0 \cdot 0096\end{array}$ & $\begin{array}{l}0.0256 \\
0.0665\end{array}$ & $\begin{array}{l}0.44 \\
0.98\end{array}$ & $\begin{array}{l}1 \cdot 89 \\
3.6 \mathrm{r}\end{array}$ \\
\hline
\end{tabular}

$* *=0 \cdot 0$ I level of significance.

variation between dates of measurements was usually highly significant. This variation was presumably due to minute differences in the medium and its preparation, slight fluctuations in temperature or both. Age of cultures was not a contributing factor since two-week-old conidia were used for both dates.

The variation among progeny from both crosses was highly significant. Based upon the estimates of $k$, one could conclude that $2 \mathrm{I} \mathrm{A}$ and 26a differ by at least two genes. One of these genes controls the pattern of growth with the pleiotropic effect of this locus influencing growth rate by approximately $0.3 \mathrm{~mm} . / \mathrm{hr}$. The other gene(s) would have a considerably smaller effect upon growth rate. Cultures 2 I A and I5 a presumably differ by at least three to four genes. Again, the gene controlling the pattern of growth has a large effect upon rate of growth. From the overlapping distributions, based on pattern of growth and shown in fig. 4, one could conclude that at least some of the remaining genes involved in this difference are not linked to the growth pattern locus.

\section{DISCUSSION}

Without the results from reverse selection in the cross $\mathrm{Hon}_{3} \mathrm{~A} / 77 \mathrm{a}$, as shown in fig. 2, one might have been inclined to believe that selection for fast growth rate had resulted in a genotypically stable line. There had been no apparent increase in growth rate in the last ro cycles of 
selection, nor in the eight cycles involving the fast $\times$ slow selection line. In addition, the phenotypic variances for each cycle over this period were very small and quite similar in magnitude. Furthermore, a relatively early approach to genetic fixation might have been predicted due to the intensity of inbreeding. The response to reverse selection commencing at cycle II, however, indicated that genetic variability still existed in the selected line and that this line had not become genotypically fixed.

Although genetic variability still existed in the selected line, it presumably was not of a kind to permit the growth rate to exceed a particular level. Similar observations were made by Lee and Pateman (I96I) after I5 generations of selection for increased ascospore length in Neurospora. They found that although genetic variability still existed, it was neither sufficient to allow for additional increases in length nor for an effective response to reverse selection. Natural selection, in the form of a fertility barrier, was opposing artificial selection. Only by altering their selection method, in ways that infertility problems could be overcome, were they able to increase ascospore length. Whether fertility had become the limiting factor in the present experiment is not certain. Fertility problems were encountered in some lines and resulted in the cessation of selection (Papa et al., 1966). Other lines, including the one portrayed in fig. 2, exhibited no apparent infertility problems.

To test the possibility of having reached a physiological upper limit in selecting for fast growth rate, crosses were made between selected strains of different origins. Progeny obtained following selection in crosses $77 \mathrm{a} / 74^{*}$ for 12 cycles and from Hon Ia/Hon $3 \mathrm{~A}^{*}$ for i I cycles at $35^{\circ} \mathrm{C}$. were crossed. Response to selection had essentially ceased in both lines. Following six generations of selection, growth rate had increased beyond the previous limit $(6 \cdot \mathrm{I} 2 \mathrm{~mm}$. $/ \mathrm{hr}$.) obtained by selection in Hon Ia/Hon $3 \mathrm{~A}$, to $6.35 \mathrm{~mm}$./hr. There was no indication that selection limits had been reached. Additional generations of selection are needed to determine the possible extent of selection responses in this new line. It can be concluded, however, that a physiological limit had not been reached previously. The improved growth rate presumably occurred as a result of combining different favourable alleles for growth from different sources.

Additional selection advances in the line portrayed in fig. 2 might require breaking up rather tight linkages to get recombinant individuals exhibiting superior growth rates. For example, the retention of a certain amount of genetic variability might be expected among genes closely linked to the mating type locus. Since opposite mating types are required for crosses, gene complexes closely linked to each mating type and having an effect on growth rate could be maintained. Breaking up these linkages might permit the recovery of recombinants

* 74A, $77 \mathrm{a}$ and Hon Ia,-3A are standard St Lawrence and Honduras strains of $\mathcal{N}$. crassa, respectively. 
possessing increased growth rates. Thus, to obtain favourable recombinations within particular chromosome segments might require a considerably larger number of generations of selection than was included in the present study.

While it is realised that breaking up certain tight linkages might permit the recovery of favourable genotypes for fast growth rate, these same linkages, if broken, could provide the basis for response to reverse selection. It is extremely unlikely, however, that the response to reverse selection in the present experiment could be accounted for in this manner.

Mutation may have accounted for some of the response to reverse selection. A morphological change was noted in the second generation of reverse selection and could be attributed to a single gene mutation. This morphological variant was associated with an approximately $0.3 \mathrm{~mm}$./ hr. decrease in growth rate. Whether this situation represented linkage of a major gene to part of a polygenic system affecting growth rate is not certain. Unless recombination between the polygenic system and the growth pattern locus can be demonstrated, it would appear most likely that the effect on growth rate is one of the pleiotropic effects of this locus. This latter conclusion would seem to be the most logical since the dense growth has obviously resulted from the production of more aerial hyphæ than are produced in faster growing lines with sparser growth. Thus, the allele responsible for dense growth has given the organism the capacity to produce a luxuriant growth pattern, but only at the expense of horizontal growth. If one were to compare the two strains on the basis of weight of mycelia produced in a given time, the difference might not be significant.

Estimates of the number of genes involved between forward- and reverse-selected lines indicated that relatively few genes could have large effects upon growth rate. A similar finding was reported by Croft and Simchen (1965) in growth rate studies of monocaryons of Collybia velutipes. In two cases they were able to demonstrate major effects on growth rate by single genes controlling morphological differences.

Although mutation may have accounted for some of the response to reverse selection, it is improbable that each step-wise decrease in growth rate was due to a new mutation. Apparently, other genetic phenomena were involved in at least part of the observed response. Simchen (1966) points out that unidirectional non-additive interactions and the instability of certain genotypes are probably quite important in haploid polygenic systems. While neither of these possibilities was investigated in the present experiment, other selection results in Neurospora (Papa, 1964) indicate that pronounced changes in stability could occur during the course of selection. The genetic basis of this instability was not examined.

No attempt was made in the present study to locate polygenic activity controlling growth rate. Using appropriate markers, however, 
it should be possible to acquire information of this nature. Lee and Pateman (I959, I96I) established linkage between part of the polygenic system controlling ascospore length and the albino locus in Neurospora. Similarly, Simchen ( 1966 ) demonstrated linkage of a polygenic block affecting growth rate in Schizophyllum commune to the A incompatibility factor. Some of the most extensive studies on the location of polygenes have involved bristle numbers in Drosophila (Mather, I94I, I 942; Wigan, I949; Breese and Mather, I 957). Thoday (I96I) has outlined a procedure for locating polygenic activity.

\section{SUMMARY}

I. Responses to selection for fast growth rate among the progeny of a cross between two strains of Neurospora " plateaued " after approximately nine generations. No further increases in growth rate were noted for Io additional generations of selection.

2. Reverse selection, following I I generations of forward selection, was effective in reducing growth rate. Apparently, potential genetic variability was freed, thus permitting response in the reverse direction.

3. Analyses of the growth rates of progeny from crosses between forward- and reverse-selected lines demonstrated the segregation of some of the genes controlling growth rate. One gene controlling a morphological difference had a major effect upon growth rate.

Acknowledgments.-We acknowledge receipt of financial support for this work from the Office of Naval Research through Contract No. Nonr-40I (39), Project No. (NR 042-2 I2) and in part from grant GM I 2953 National Institutes of Health, U.S. Public Health Service. K. E. P. acknowledges receipt of a traineeship from the National Institute of General Medical Sciences, NIH, Grant TI GM 1035.

The authors express their sincere thanks to Professor A. A. Fleming for his critical reading of the manuscript and to Mr Ronnie Morrison for skillfully inking and lettering the figures.

\section{REFERENCES}

BEAdLe, G. W., AND TAtum, E. L. I945. Neurospora II. Methods of producing and detecting mutations concerned with nutritional requirements. Amer. F. Bot., 32, 678-686.

BREESE, E. L., AND MATHER, K. I957. The organization of polygenic activity within a chromosome in Drosophila. I. Hair characters. Heredity, II, 372-395.

CLAYTON, G. A., AND ROBERTSON, ALAN. 1957. An experimental check on quantitative genetical theory. II. The long-term effects of selection. J. Genet., 55, $15^{2-1} 70$.

CROFT, J. H., AND SIMCHEN, G. I965. Natural variation among monokaryons of Collybia velutipes. Amer. Natur., 99, 45 I-462.

FALCONER, D. S. I955. Patterns of response in selection experiments with mice. Cold Spr. Harb. Sym. Quant. Biol., 20, 1 78-196.

FALCONER, D. S., AND KING, J. W. B. I953. A study of selection limits in the mouse. J. Genet., 5I, 56I-58I.

federer, w. T. I96r. Experimental error rates. Proc. Amer. Soc. Hort. Sci., 78, $605-6$ I 5 .

FEDERER, W. T., PAPA, K. E., AND SRB, A. M. 1966. Selection models for a quantitative trait in Neurospora. Biometrics (manuscript in process of publication). 
FRASER, A. S. 1957. II. Effects of linkage on rates of advance under selection. Austr. J. Biol. Sci. Io, 492-499.

FRASER, A. S. 196o. Simulation of genetic systems by automatic digital computers. Biometrical Genetics, pp. 70-83. Pergamon Press.

LEE, в. T. O., AND PATEMAN, J. A. 1959. Linkage of polygenes controlling size of ascospore in Neurospora crassa. Nature, 183, 698-699.

LeE, B. T. o., AND pateman, J. A. 1961. Studies concerning the inheritance of ascospore length in Neurospora crassa. I. Studies on large-spored strains. Austr. 7. Biol. Sci., I4, 223-230.

MATHER, к. 1941. Variation and selection of polygenic characters. 7. Genet., $4^{I}$, 159-193.

MATHER, к. 1942. The balance of polygenic combinations. 7. Genet., 43, 311-336.

Mather, K. 1943. Polygenic inheritance and natural selection. Biol. Reviewe, 18, $32-64$.

MATHER, K. 1949. Biometrical Genetics. Methuen, London.

PAPA, K. E. 1964. Effectiveness of selection based on variability uncomplicated by heterotic effects in Neurospora. ONR Technical Report No. 18, Contract No. Nonr-409(39), Project No. (NR o42-2 I2), Cornell University.

PAPA, K. E., SRB, A. M., AND FEDERER, W. T. 1966. Selection for increased growth rate in inter- and intra-strain crosses of Neurospora. Heredity 2I, 59-613.

robertson, alan. 1955. Selection in animals: Synthesis. Cold Spr. Harb. Sym. Quant. Biol., 20, 225-229.

RYAN, F. J., BEADLE, G. W., AND TATUM, E. L. 1943. The tube method of measuring the growth rate of Neurospora. Amer. F. Bot., 3o, 784-799.

SIMCHEN, G. 1966. Monokaryotic variation and haploid selection in Schizophyllum commune. Heredity, 21, 241-263.

THODAY, J. M. I96I. Location of polygenes. Nature, I9I, 368-370.

WIGAN, L. G. 1949. The distribution of polygenic activity on the $\mathrm{x}$-chromosome of Drosophila melanogaster. Heredity, 3, 53-66. 